\title{
The novel MAPT mutation K298E: mechanisms of mutant tau toxicity, brain pathology and tau expression in induced fibroblast-derived neurons
}

\author{
Mariangela Iovino • Ulrich Pfisterer • Janice L. Holton • Tammaryn Lashley • \\ Robert J. Swingler $\cdot$ Laura Calo $\cdot$ Rebecca Treacy $\cdot$ Tamas Revesz $\cdot$ Malin Parmar • \\ Michel Goedert $\cdot$ Miratul M. K. Muqit $\cdot$ Maria Grazia Spillantini
}

Received: 17 May 2013 / Accepted: 15 November 2013 / Published online: 30 November 2013

(c) The Author(s) 2013. This article is published with open access at Springerlink.com

\begin{abstract}
Frontotemporal lobar degeneration (FTLD) consists of a group of neurodegenerative diseases characterized by behavioural and executive impairment, language disorders and motor dysfunction. About 20-30\% of cases are inherited in a dominant manner. Mutations in the microtubule-associated protein tau gene (MAPT) cause frontotemporal dementia and parkinsonism linked to chromosome 17 (FTDP-17T). Here we report a novel MAPT mutation (K298E) in exon 10 in a patient with FTDP-17T. Neuropathological studies of post-mortem brain showed widespread neuronal loss and gliosis and abundant deposition of hyperphosphorylated tau in neurons and glia. Molecular studies demonstrated that the K298E mutation affects both protein function and alternative mRNA splicing. Fibroblasts from a skin biopsy of the proband taken at post-mortem were directly induced into neurons (iNs) and expressed both 3-repeat and 4-repeat tau isoforms. As well as contributing new knowledge on MAPT mutations in
\end{abstract}

M. Iovino $\cdot$ L. Calo $\cdot$ M. G. Spillantini $(\bowtie)$

Cambridge Centre for Brain Repair, University of Cambridge,

Cambridge, UK

e-mail:mgs11@cam.ac.uk

\section{U. Pfisterer · M. Parmar}

Department of Experimental Medical Science and Lund Stem

Cell Center, Lund University, Lund, Sweden

J. L. Holton · T. Lashley · T. Revesz

Queen Square Brain Bank for Neurological Disorders,

Department of Molecular Neuroscience, Institute of Neurology,

University College London, London, UK

\section{R. J. Swingler · M. M. K. Muqit}

Department of Neurology, Ninewells Hospital, College

of Medicine, Nursing and Dentistry, University of Dundee,

Dundee, UK
FTDP-17T, this is the first example of the successful generation of iNs from skin cells retrieved post-mortem.

Keywords K298E MAPT mutation · Tauopathies · Human induced-neurons

\section{Introduction}

Frontotemporal lobar degeneration (FTLD) consists of a group of disorders that manifest as the result of an initial pathological damage of the frontal and temporal lobes of the cerebrum $[2,19,21]$. Hereditary forms represent $20-30 \%$ of all cases of FTLD and up to $15-20 \%$ of these carry mutations in $M A P T$, the gene coding for the microtubule-associated protein tau [22]. Patients carrying MAPT mutations are characterized by filamentous and hyperphosphorylated tau inclusions in neuronal and glial

\section{R. Treacy}

Genetics Laboratories, Molecular Genetics, Addenbrooke's Treatment Centre, Addenbrooke's Hospital, Cambridge, UK

M. Goedert

Medical Research Council, Laboratory of Molecular Biology, Cambridge, UK

M. M. K. Muqit ( $\square)$

Medical Research Council Protein Phosphorylation and Ubiquitylation Unit, College of Life Sciences, University of Dundee, Dundee, UK

e-mail: m.muqit@dundee.ac.uk 
cells, causing neurodegeneration. In normal brain, tau promotes microtubule assembly and stabilization, and is involved in axonal transport [10]. In adult human brain six tau isoforms are produced by alternative mRNA splicing from a single gene located on chromosome 17 [7]. They differ by the presence of 29- or 58- amino acid inserts in the amino-terminal half of the protein, and by the presence of three (3R) or four (4R) repeats in the carboxylterminal half. The repeats and some adjoining sequences constitute the microtubule-binding domains of tau [12]. $M A P T$ mutations have their primary effect either at the protein level, reducing the ability of tau to interact with microtubules, or at the mRNA level, altering the balance between $3 R$ and $4 R$ tau isoforms $[6,10]$. Here we report the identification and characterization of a novel MAPT mutation in exon 10,892 AAA $>$ GAA, leading to a change from lysine to glutamate at codon 298 (K298E). This mutation significantly reduced the ability of tau to promote microtubule assembly and altered exon 10 splicing by increasing the expression of 4R tau. In the proband's brain, neuronal and glial $4 \mathrm{R}$ tau pathology was present. Moreover induced neurons (iNs) obtained via transcription-factor mediated direct conversion from patient skinderived fibroblasts with the K298E mutation expressed both $3 R$ and $4 R$ tau isoforms. This expression pattern was similar to that of neurons from an age-matched control, but differed from that of neurons obtained from embryoderived human fibroblasts.

\section{Materials and methods}

\section{Case report}

The proband was referred at the age of 67 because of progressive gait difficulties and non-fluent aphasia. Her symptoms had rapidly progressed over the preceding 2 years. Her father had been diagnosed with Alzheimer's disease in his $60 \mathrm{~s}$ and had died at the age of 67 . The only living relative is the proband's son who is currently asymptomatic. The proband died at the age of 68 and her brain was donated to the Queen Square Brain Bank for Neurological Disorders, UCL Institute of Neurology.

\section{Genetic analyses}

Genomic DNA was extracted from $5 \mathrm{ml}$ of blood collected from the patient and it was analysed at the East Anglian Regional Molecular Genetics Laboratory, Addenbrooke's Hospital, Cambridge UK. Fluorescence sequencing of exons 1-5, 7, 9-13 (including intronic boundaries) of MAPT gene was performed.
Purification of recombinant tau

The quick change lightning site-directed mutagenesis kit (Stratagene, Edinburgh, UK) was used to introduce the K298E mutation into the shortest 4R isoform (4R0N, T43) of human brain tau.

Wild-type, P301S and K298E mutant human tau cDNAs were subcloned into pRK172 and transformed into BL21 (DE3) E. coli, as described [8]. Briefly, following induction with $0.4 \mathrm{mM}$ IPTG, the cells were collected by centrifugation, the pellet was resuspended in $30 \mathrm{ml}$ of $25 \mathrm{mM}$ Tris $\mathrm{pH}$ 7.4, $10 \mathrm{mM}$ EDTA, $0.1 \mathrm{mM}$ DTT, $0.1 \mathrm{mM}$ PMSF and the cell suspension disrupted at $4{ }^{\circ} \mathrm{C}$ in a constant system cell disruptor. Recombinant tau protein was purified by anionexchange chromatography and dialysed overnight at $4{ }^{\circ} \mathrm{C}$ against $40 \mathrm{mM}$ HEPES, pH 7.4, plus $0.1 \mathrm{mM}$ DTT.

Microtubule assembly assay

The ability of recombinant tau proteins to promote microtubule assembly was monitored by turbidity, as described [8]. Tubulin (Cytoskeleton Inc., Denver, CO, USA) was dissolved in G-PEM buffer (80 mM Na-PIPES pH 6.9, $1 \mathrm{mM} \mathrm{MgCl} 2,1 \mathrm{mM}$ EGTA, $1 \mathrm{mM} \mathrm{GTP}$ ) and mixed with $77.5 \mu \mathrm{l}$ of G-PEM. Wild-type or mutant tau was then added and the mixture was quickly transferred to a quartz cuvette. The final concentrations of both tubulin and tau were $1 \mathrm{mg} / \mathrm{ml}$. Polymerization of microtubules was monitored by measuring the absorbance at $350 \mathrm{~nm}$ at $37{ }^{\circ} \mathrm{C}$ over $10 \mathrm{~min}$. All experiments were performed in triplicate.

Minigene transfection

A previously described [1] MAPT minigene construct encompassing exons 9-11 and flanking E10 intronic sequences in pcDNA3 was kindly provided by $\mathrm{Dr}$ R. Vuono, as was the construct with the $\mathrm{I} 10+3 \mathrm{G}>\mathrm{A}$ intronic mutation. The quick change lightning site-directed mutagenesis kit was used to introduce the K298E mutation in exon 10 of MAPT. Neural stem cells (NSCs) were obtained from human embryonic stem cells and maintained in a neural precursor stage in defined medium in the presence of $20 \mathrm{ng} / \mathrm{ml}$ of FGF2, as described [15, 24]. Transient transfections were performed using Lipofectamine 2000, according to the manufacturer's instructions (Invitrogen, Eugene OR, USA). Cells were plated into a six-well plate at a density of $2 \times 10^{5}$ cells/well and incubated in the absence of antibiotics for $24 \mathrm{~h}$. For single transfections, $2 \mu \mathrm{g}$ of the wild-type or mutant minigene DNA was mixed with $10 \mu 1$ Lipofectamine 2000 in Optimem medium (Invitrogen, Eugene OR, USA) and incubated for $20 \mathrm{~min}$ at room 
temperature. For co-transfections, $1 \mu \mathrm{g}$ of each construct (wild-type and mutant) was used. Cells were exposed to the transfection mixture for $5 \mathrm{~h}$ at $37{ }^{\circ} \mathrm{C}$ and $5 \% \mathrm{CO}_{2}$ and returned to their growing medium. To analyse minigene expression, cells were harvested $48 \mathrm{~h}$ after transfection.

\section{RNA extraction and RT-PCR}

Total RNA was extracted from NSCs with the RNeasy mini kit (Qiagen, Crawley, UK). RNA concentration and purity were estimated using spectrophotometry (absorbance at 230, 260 and $280 \mathrm{~nm}$ ). Three-repeat and $4 \mathrm{R}$ tau mRNA expression was detected by RT-PCR using the onestep RTPCR kit (Qiagen, Crawley, UK) and the following primers: exon 9 forward $5^{\prime}$ CTCCAAAATCAGGGGATCGC $3^{\prime}$ and exon11 reverse $5^{\prime}$ TATGATGGATGTTGCCTAATGAGCC $3^{\prime}$. Bands corresponding to mRNAs with and without exon 10 were separated by electrophoresis on $1 \%$ agarose gels. The same method was used for RT-PCR of RNA extracted from frontal cortex of the patient with the K298E mutation and of a control subject.

Human brain tissues

Tissue from cerebral cortex of a patient with the $\mathrm{I} 10+3$ G>A MAPT mutation was obtained from the Brain Bank of the Indiana University Alzheimer Centre [26]. Frontal cortex from an age-matched control subject was obtained from the Cambridge Hospital Brain Bank. The work on human tissue was covered by Cambridge LREC ethical

Table 1 Antibodies used in this study

\begin{tabular}{lllll}
\hline Antibody & Epitope/antigen & Species & Dilution & Source \\
\hline AT8 & Tau (phospho-Ser202/Ser205) & Mouse & $1: 600$ & Autogen Bioclear, Wiltshire, UK \\
RD03 & 3-Repeat tau & Mouse & $1: 2,000$ & Dr Rohan De Silva/Millipore \\
RD04 & 4-Repeat tau & Mouse & $1: 100$ & Dr Rohan De Silva/Millipore \\
AT100 & Tau (phospho Ser212/Thr214/T217) & Mouse & $1: 200$ & Autogen Bioclear, Wiltshire, UK \\
A $\beta$ & $\beta$-amyloid protein (M0872) & Mouse & $1: 100$ & DAKO, Ely, UK \\
$\alpha$-Synuclein & $\alpha$-Synuclein & Mouse & $1: 75$ & Novocastra, Peterborough, UK \\
p62 & p62 lck ligand & Mouse & $1: 100$ & BD Transduction Labs, Oxford, UK \\
TDP-43 & Tar DNA-binding protein-43 & Mouse & $1: 800$ & Abnova, Taipei City, Taiwan \\
GFAP & Glial fibrillary acidic protein & Rabbit & $1: 1 \mathrm{~K}$ & DAKO, Ely, Uk \\
Neurofilaments & Phosphorylated (SMI 31) and non- & Mouse & SMI 31-1:5,000 & Sternberger Monoclonals Inc, Baltimore, USA \\
& phosphorylated (SMI 32) neurofilaments & & SMI 32-1:500 & \\
$\alpha$ B crystallin & Novacastra & Mouse & $1: 300$ & Novocastra, Peterborough, UK \\
\hline
\end{tabular}

Fig. 1 Microtubule assembly assay. Polymerization of tubulin induced by wild-type tau, P301S tau and K298E tau. Microtubule assembly was monitored over time by optical density (absorbance $350 \mathrm{~nm}$ ). The results are expressed as the means of three different experiments

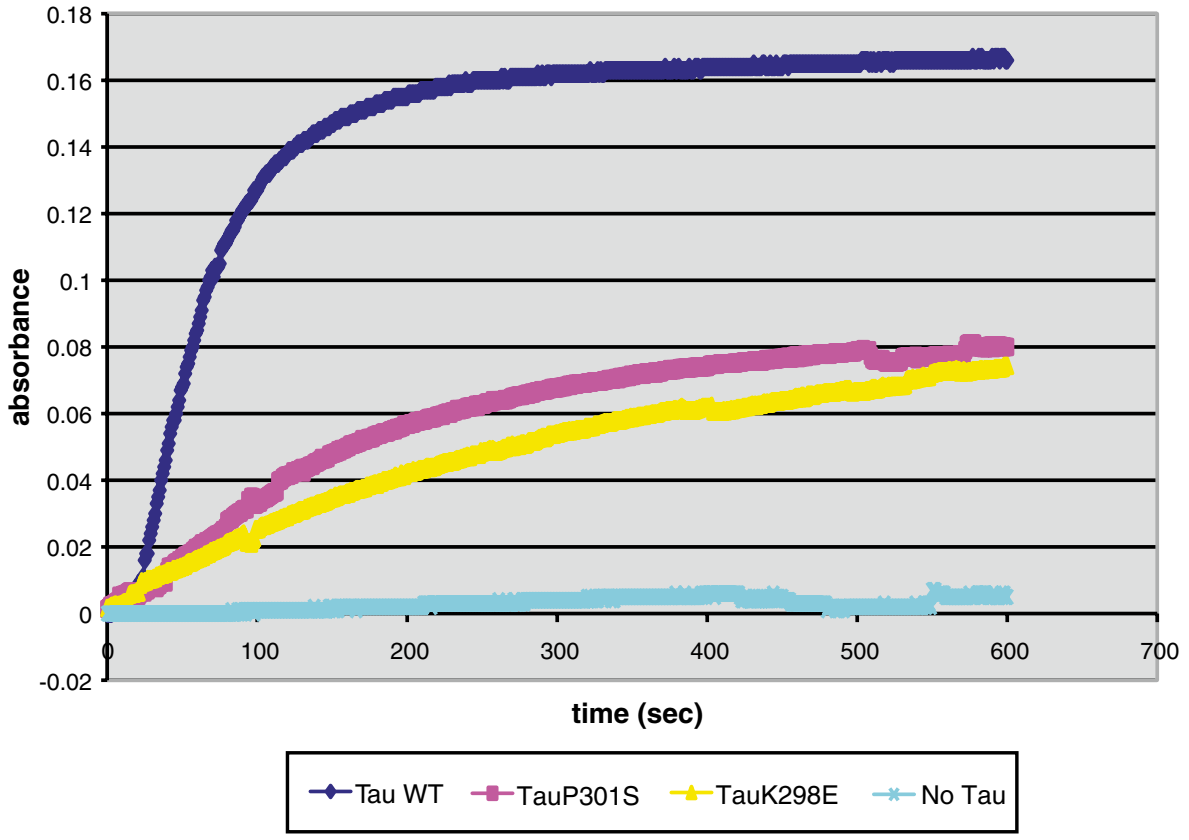


approval (ref no 09/40). Neuropathological investigation was performed at the Queen Square Brain Bank for Neurological Disorders, London. Handling of human tissue was according to the UK Human Tissue Act 2006. Approval for "Generation of patient-specific stem cells for research in neurodegenerative disorders of the CNS" was granted by the Hertfordshire Research Ethics Committee (ref no 09/ H0311/88).

\section{Immunohistochemistry}

Following fixation in $10 \%$ buffered formalin, the left half of the proband's brain was sliced in the coronal plane and blocks were selected for histological examination. The right half of the brain was sliced in the coronal plane, flash frozen and stored at $-80{ }^{\circ} \mathrm{C}$. Paraffin-embedded sections $(8 \mu \mathrm{m})$ from cortical, subcortical, brainstem and cerebellar regions were stained using haematoxylin eosin (HE) and Gallyas silver impregnation. Immunohistochemistry was performed using the antibodies listed in Table 1. Sections were deparaffinised using xylene and rehydrated using graded alcohols. Endogenous peroxidase activity was blocked with methanol/0.3 \% $\mathrm{H}_{2} \mathrm{O}_{2}$. Antigen retrieval was achieved by heating sections in a pressure cooker for 10 min in citrate buffer ( $\mathrm{pH}$ 6.0). For $\mathrm{A} \beta$ and $\alpha$-synuclein immunohistochemistry, sections required pre-treatment with formic acid for $10 \mathrm{~min}$ prior to antigen retrieval. Sections were incubated in dried milk solution $(10 \%$ in PBS, $30 \mathrm{~min})$ to block non-specific binding. Primary antibodies diluted in PBS were applied for $60 \mathrm{~min}$ at room temperature, followed by either biotinylated anti-rabbit IgG or biotinylated anti-mouse IgG (1:200, $30 \mathrm{~min}$; DAKO) and ABC complex (30 min; DAKO). The sections were developed with di-aminobenzidine $/ \mathrm{H}_{2} \mathrm{O}_{2}$ and counterstained with HE.

Neuronal loss and gliosis were assessed on HE-stained sections and scored semi-quantitatively as follows: $-=$ none $;=$ mild $;++=$ moderate $;+++=$ severe . The frequency of tau-positive inclusions of different types was assessed using antibody AT8 and scored semi-quantitatively: $-=$ none $;+=$ infrequent $++=$ moderate; $+++=$ frequent.

\section{Immunocytochemistry}

Cells were fixed with $4 \%$ paraformaldehyde and preincubated for $1 \mathrm{~h}$ in blocking solution (5\% normal goat serum, $0.02 \%$ Tween in PBS). Primary antibodies (monoclonal 3R tau isoform 1:500, Millipore; monoclonal 4R tau isoform, 1:500 Millipore; polyclonal betalIItubulin 1:1,000, Covance) were incubated overnight at $4{ }^{\circ} \mathrm{C}$. For $3 \mathrm{R}$ and $4 \mathrm{R}$ tau immunofluorescence, cells were incubated with biotinylated secondary antibodies $(1: 250)$ for $2 \mathrm{~h}$ at room temperature and $1 \mathrm{~h}$ with fluorescent streptavidin (Alexa Invitrogen, 1:500). Anti rabbit fluorescent-conjugated secondary antibodies (Alexa, Invitrogen Molecular Probes) diluted 1:1,000 were used for betallItubulin staining. Double-labelling immunofluorescence was performed by sequential incubation with the appropriate primary

a
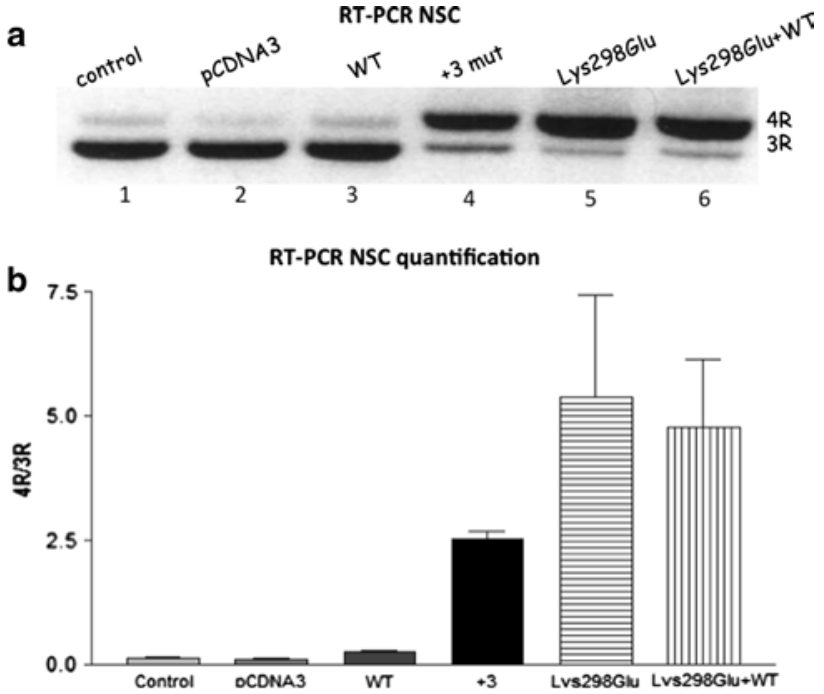

C

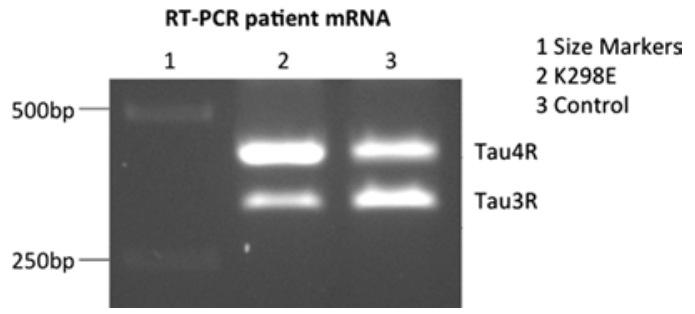

Fig. 2 Reverse transcription polymerase chain reaction (RT-PCR) analysis of tau mRNA expression in human neural stem cells transfected with a tau minigene and mRNA extracted from frontal cortex of the K298E patient. The tau minigene construct consisted of the pcDNA3 vector containing tau exons 9,10 and 11, and the E10 flanking intronic sequences of minimal length for correct splicing. The splicing patterns of MAPT exon 10 in minigene transfected human neural stem cells (NSCs) are shown. a Untransfected human NSCs expressed high levels of 3R tau mRNA and low levels of $4 R$ tau mRNA (lane 1) similar to cells transfected with the empty pcDNA3 vector (lane 2). Transfection with the wild-type tau minigene did not change the ratio between the two mRNAs (lane 3), with the expression level of $3 \mathrm{R}$ tau mRNA remaining predominant. When human NSCs were transfected with the minigene carrying the +3 MAPT mutation, the mRNA level of $4 \mathrm{R}$ tau increased (lane 4) and this also occurred after transfection with the minigene carrying the K298E mutation (lane 5). Co-transfection of wild-type and K298E minigenes did not alter the ratio between $3 \mathrm{R}$ and $4 \mathrm{R}$ tau isoforms mRNAs (compared to K298E alone), indicating that the K298E mutation has a dominant effect on exon 10 splicing (lane 6). The quantification shown in $\mathbf{b}$ was the result of three different transfection experiments and was performed using the Image $\mathbf{J}$ program (http://imagej.nih. gov/). c RT-PCR of mRNA extracted from frontal cortex of the K298E mutation patient showed a clear increase in 4R tau mRNA expression and a decrease in $3 \mathrm{R}$ tau mRNA expression compared to mRNA extracted from an age-matched control subject 
antibodies. Cell nuclei were visualized with Hoechstbye (Sigma Aldrich, 1:5,000) as previously reported [15]. Fluorescence was visualized using a Leica TMDM 6000B fluorescence microscope.
Immunoblotting

Soluble tau was extracted with perchloric acid as described [8] from the frontal cortex of the proband, from the frontal

Table 2 Semi-quantitative assessment and regional distribution of neuronal loss, gliosis and tau pathology

\begin{tabular}{|c|c|c|c|c|c|c|c|c|c|c|}
\hline & \multirow{2}{*}{$\begin{array}{l}\text { Neuronal } \\
\text { loss }\end{array}$} & \multirow[t]{2}{*}{ Gliosis } & \multicolumn{8}{|c|}{ Tau pathology } \\
\hline & & & Threads & Dots & $\begin{array}{l}\text { Neuronal } \\
\text { rings/crescents }\end{array}$ & $\begin{array}{l}\text { Neuronal } \\
\text { flame shaped }\end{array}$ & $\begin{array}{l}\text { Neuronal } \\
\text { globular }\end{array}$ & $\begin{array}{l}\text { Neuronal } \\
\text { diffuse }\end{array}$ & Oligodendroglial & Astrocytic \\
\hline \multicolumn{11}{|l|}{ Cortex } \\
\hline Frontal & ++ & +++ & +++ & + & +++ & + & ++ & ++ & + & ++ \\
\hline Motor & + & ++ & +++ & + & +++ & ++ & + & +++ & + & + \\
\hline Temporal & + & + & +++ & + & +++ & + & + & ++ & + & ++ \\
\hline Parietal & ++ & +++ & +++ & + & +++ & + & ++ & ++ & + & ++ \\
\hline Occipital & - & + & ++ & + & + & ++ & + & + & - & + \\
\hline Cingulate & + & ++ & +++ & + & +++ & + & + & +++ & & + \\
\hline \multicolumn{11}{|l|}{ Sub-cortical white matter } \\
\hline Frontal & N/A & +++ & ++ & ++ & N/A & N/A & N/A & N/A & +++ & - \\
\hline Motor & N/A & +++ & ++ & + & N/A & N/A & N/A & N/A & +++ & - \\
\hline Temporal & N/A & + & ++ & ++ & N/A & N/A & N/A & N/A & +++ & - \\
\hline Parietal & N/A & +++ & ++ & ++ & N/A & N/A & N/A & N/A & +++ & - \\
\hline Occipital & N/A & ++ & + & + & N/A & N/A & N/A & N/A & + & - \\
\hline Cingulate & N/A & ++ & ++ & ++ & N/A & N/A & N/A & N/A & +++ & - \\
\hline Amygdala & + & +++ & ++ & +++ & +++ & + & ++ & +++ & + & ++ \\
\hline \multicolumn{11}{|l|}{ Hippocampus } \\
\hline Dentate fascia & $\begin{array}{l}- \\
\text { (Dispersed) }\end{array}$ & + & ++ & + & ++ & + & - & +++ & - & - \\
\hline CA4 & +++ & +++ & +++ & + & + & - & - & + & + & - \\
\hline CA3 & +++ & +++ & +++ & + & - & - & - & + & + & - \\
\hline CA2 & - & ++ & +++ & + & - & - & + & +++ & - & - \\
\hline CA1 & + & ++ & +++ & + & + & ++ & + & +++ & + & - \\
\hline Subiculum & + & ++ & +++ & + & + & + & ++ & +++ & + & + \\
\hline Entorhinal cortex & + & +++ & +++ & ++ & +++ & + & + & +++ & + & + \\
\hline Transentorhinal cortex & + & +++ & +++ & ++ & +++ & - & ++ & +++ & + & + \\
\hline Caudate & ++ & ++ & +++ & + & +++ & - & ++ & ++ & + & ++ \\
\hline Putamen & - & ++ & +++ & + & +++ & - & ++ & + & + & ++ \\
\hline Globus pallidus & - & +++ & ++ & + & + & - & + & + & +++ & - \\
\hline Meynert & - & ++ & +++ & ++ & - & - & +++ & +++ & + & + \\
\hline Thalamus & - & ++ & +++ & + & - & - & + & ++ & +++ & + \\
\hline Subthalamic nucleus & - & ++ & ++ & + & - & - & ++ & ++ & + & - \\
\hline Midbrain tegmentum & - & ++ & ++ & + & + & - & + & +++ & + & - \\
\hline Substantia nigra & + & ++ & +++ & + & + & - & + & +++ & + & - \\
\hline Locus coeruleus & + & ++ & + & ++ & - & - & - & ++ & - & - \\
\hline Pontine tegmentum & - & ++ & ++ & ++ & - & - & + & +++ & + & - \\
\hline Pontine nuclei & - & ++ & + & +++ & - & - & + & ++ & ++ & - \\
\hline $\begin{array}{l}\text { Dorsal motor nucleus } \\
\text { of vagus }\end{array}$ & - & ++ & + & + & - & - & + & + & - & - \\
\hline Twelfth nerve nucleus & - & ++ & + & ++ & - & - & - & - & + & - \\
\hline Inferior olive & - & ++ & - & +++ & - & - & - & - & - & + \\
\hline Cerebellar Purkinje cells & + & + & - & + & - & - & - & - & - & - \\
\hline Cerebellar white matter & N/A & ++ & - & + & N/A & N/A & N/A & N/A & - & - \\
\hline Dentate nucleus & - & + & + & + & - & - & + & + & - & - \\
\hline
\end{tabular}

N/A not applicable 
cortex of a patient with the $\mathrm{I} 10+3 \mathrm{G}>\mathrm{A}$ intronic $M A P T$ mutation [26] and from the frontal cortex of a control subject. Insoluble tau was extracted with sarkosyl from the frontal cortex of the proband, as described [9]. Soluble and sarkosyl-insoluble tau proteins were dephosphorylated with $0.3 \mathrm{U} / \mu 1$ alkaline phosphatase from $E$. coli (SigmaAldrich) in $50 \mathrm{mM}$ Tris- $\mathrm{HCl}$ and $5 \mathrm{mM} \mathrm{MgCl}_{2}$ for $4 \mathrm{~h}$ at $65{ }^{\circ} \mathrm{C}$. The proteins were separated on $10 \%$ SDS-PAGE and transferred onto immunobilon-P (Millipore, Bedford, MA, USA). The membranes were incubated for $1 \mathrm{~h}$ with $5 \%$ milk w/v (Premier International Foods, Spalding, UK) in TBS/0.01\% Tween-20. They were then probed with a polyclonal anti-human tau serum (Dako, Glostrup, Denmark, 1:1,000), followed by peroxidase-conjugated secondary antibody (Dako, 1:10,000). The blots were developed using Western lightning chemiluminescence reagent Plus (Perkin Elmer, Waltham, MA, USA).

Derivation and transdifferentiation of human fibroblasts

A skin biopsy was taken from the proband $2 \mathrm{~h}$ after death. It was cultured as an explant in DMEM supplemented with $10 \%$ FCS and $1 \%$ penicillin/streptomycin for 2 weeks until fibroblasts started to appear. The fibroblasts were then expanded and transduced during their second passage as described previously [25] with the addition of delaying doxycycline administration (=transgene activation) by 5 days. As controls we used human embryonic fibroblasts (hEFs) and human adult fibroblasts (hAFs) transduced in the same way.

\section{Results}

Patient clinical data

In 2009, a 67-year-old lady was referred to the neurology service for investigation of progressive gait difficulties and non-fluent aphasia. She had previously developed a tremor of her right hand at the age of 48, which had been non-troubling. At the age of 65 , the tremor became more severe and also spread to involve the left hand such that she had to give up writing and also driving. At that time her MMSE score was 27/30 and a CT brain scan revealed bilateral cortical atrophy. SPECT imaging by [(123) I] FP-CIT (DaTscan) was normal. Over the next 2 years, her condition progressed and she suffered a decline in her balance and mobility with initially recurrent falls and then progressing such she could no longer stand or sit unaided. She also exhibited a decline in her speech with severe word finding difficulties. This was associated with marked anxiety and cognitive decline. She had now become dependent for all her daily living activities. During this time there had been
Fig. 3 Neuropathological features in the anterior frontal cortex of the proband. There was mild superficial spongiosis (a), ballooned neurons were identified in $\mathrm{H} \& \mathrm{E}$ stained sections (b) and were confirmed by $\alpha \mathrm{B}$ crystallin immunohistochemistry (c). A proportion of neurons contained basophilic inclusions (d). Tau-immunoreactive inclusions were abundant (e). High magnification revealed tau-positive dots and threads (f arrow and $\mathbf{g}$ arrow, respectively) and frequent neuronal inclusions with ring or crescent morphology (f), diffuse (g), flameshaped (h) or globular appearance $(\mathbf{j})$. In the subcortical white matter tau-immunoreactive oligodendroglial inclusions and threads were abundant (k). All inclusion types showed strong immunoreactivity for 4R tau isoforms (i), including structures resembling tufted astrocytes (m) and neuronal inclusions (n), while only a minority of inclusions could be identified using immunohistochemistry for 3R tau (l). There was strong immunoreactivity for p62 in a proportion of neuronal (o) and astrocytic inclusions (o inset). Many neuronal (p), astrocytic (q) and oligodendroglial inclusions ( $\mathbf{r}$ ) in addition to threads ( $\mathbf{r}$ arrow) were argyrophilic as demonstrated using the Gallyas silver impregnation method. a, b, d H\&E; c $\alpha \mathrm{B}$ crystallin immunohistochemistry; e-k phospho-tau immunohistochemistry (AT8); i, m, n 4R tau immunohistochemistry; I 3R tau immunohistochemistry; o p62 immunohistochemistry; p-r Gallyas silver impregnation. Bar in a represents $100 \mu \mathrm{m}$ in $\mathbf{a}, \mathbf{e} ; 50 \mu \mathrm{m}$ in $\mathbf{i}, \mathbf{l} ; 25 \mu \mathrm{m}$ in $\mathbf{b}, \mathbf{c}, \mathbf{m}-\mathbf{p} ; 10 \mu \mathrm{m}$ in $\mathbf{d}, \mathbf{f}-\mathbf{k}$, $\mathbf{q}, \mathbf{r}$

no fluctuation in her condition and no history of visual hallucinations. Her past medical history included sick sinus syndrome and she had been fitted with a cardiac pacemaker. The family history was relevant as her father was diagnosed with Alzheimer's disease in his $60 \mathrm{~s}$ and died at the age of 67 . There were no other family members with dementia. She was a non-smoker and there was no history of alcohol excess.

Significant examination findings included: an unreactive facies and severe cognitive impairment, which prevented completion of a full MMSE. There was a paucity of speech and her speech was effortful. She was able to register three objects but could not recall any. She was poor at naming objects. She could perform a one-stage command but not three. She exhibited perseveration. She had prominent primitive reflexes including grasp reflexes and rooting response. In her limbs, she had a bilateral symmetrical myoclonic tremor (arms $>$ legs), which was irregular and jerky. She had increased tone in all limbs with marked spasticity of her right arm. Her deep tendon reflexes were brisk (right $>$ left) with down-going plantar responses.

Blood investigations, including treponemal serology and vasculitic screen, were unremarkable. A repeat CT brain scan showed progression of the cerebral atrophy. CSF analysis was normal. EEG revealed intermittent slow waves, mainly in the right hemisphere, suggesting cortical dysfunction. Genetic analysis of MAPT revealed a novel single nucleotide change from $\mathrm{A}>\mathrm{G}$ (AAA to $\mathrm{AAG}$ ) at codon 298 in exon 10 which resulted in a change from Lys 298 to Glu in one allele. The proband's condition progressed and she died in 2010. 

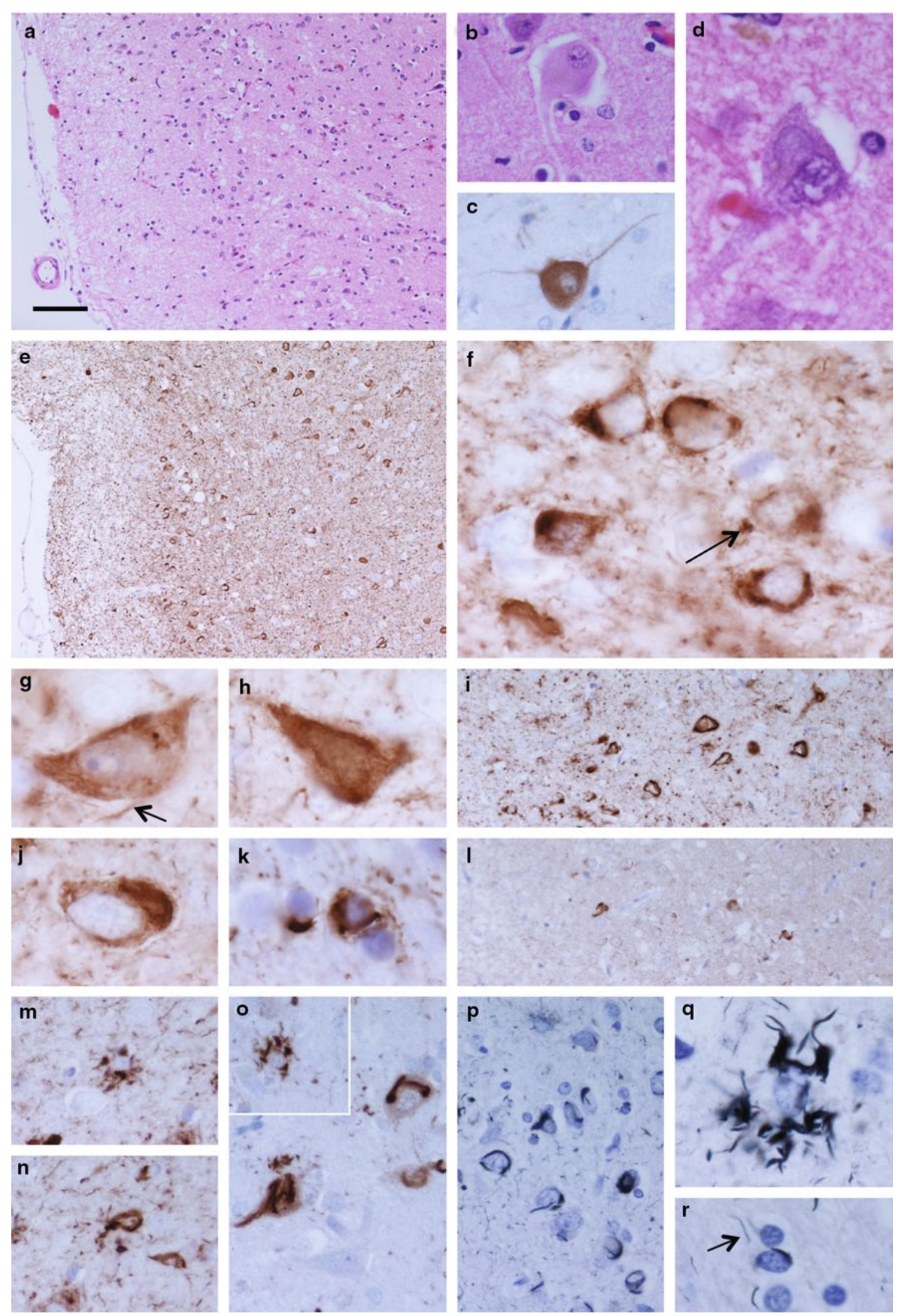
Microtubule assembly

Microtubule assembly assays were performed with recombinant wild-type or mutant tau proteins carrying the K298E mutation or the previously characterized P301S mutation. Both P301S and K298E recombinant proteins showed a reduced ability to promote microtubule assembly compared to wild-type tau (Fig. 1), suggesting that the K298E mutation disrupts the ability of tau to interact with microtubules.

\section{Tau splicing}

We next examined the effect of the K298E tau mutation on exon 10 alternative mRNA splicing in cultured cells. A minigene construct with A892>G mutation was engineered based on the previously described human tau minigene model [17]. Human NSCs were transiently transfected at day 20 of differentiation with mutant K298E or the wildtype tau minigene, as described [1]. Untransfected human NSCs expressed a strong 3R tau isoform and a very weak $4 \mathrm{R}$ tau band at the mRNA but not the protein levels as previously shown [15] (Fig. 2a, lane 1). When transfected with the empty vector or the wild-type tau minigene, the level of expression of $3 \mathrm{R}$ tau remained high. In contrast, transfection of the I10+3 mutation minigene (Fig. 2a, lane 4) or the K298E mutant minigene (Fig. 2a, lane 5) altered the ratio between $3 R$ and $4 R$ tau isoforms in favour of $4 R$ tau, indicating that the two mutations strongly reduced the splicing out of exon 10. Co-transfection of the wildtype minigene and the K298E minigene still increased the amount of transcripts with exon 10, indicating that the K298E mutation had a dominant effect on the splicing of exon 10 (Fig. 2a, lane 6). Figure 2b shows a quantification of tau isoform mRNA levels in human NSCs in three different experiments for each line. The effect of the K298E on exon 10 splicing was confirmed by RT-PCR of RNA extracted from the patient's brain. A clear increase of the $4 \mathrm{R}$ tau band was observed compared to the RT-PCR of RNA extracted from the cortex of a control subject (Fig. 2c).

\section{Brain post-mortem study}

The left half of the brain weighed $513 \mathrm{~g}$. External examination and coronal slices showed severe atrophy of the frontal lobe, including the motor strip, and the anterior temporal lobe, while the parietal and posterior temporal lobes were less severely affected and there was good preservation of the occipital lobe. There was severe dilatation of the frontal and temporal horns of the lateral ventricle. The cortical ribbon was thin throughout the frontal lobe and in all three temporal gyri anteriorly with patchy thinning in the parietal lobe and in the second and third temporal gyri posteriorly. There was atrophy of the caudate nucleus and thalamus with preservation of the putamen, globus pallidus and subthalamic nucleus. The hippocampus and amygdala were severely atrophic. Both the substantia nigra and the locus coeruleus were pale. Medulla and cerebellum were unremarkable.

\section{Histology and immunohistochemistry}

Immunohistochemistry showed widespread neuronal loss and gliosis with abundant deposition of hyperphosphorylated tau in neurons and glia. The semiquantitative assessment of these features in different brain regions is detailed in Table 2.

In addition to cortical thinning, gliosis and mild superficial spongiosis in the frontal, temporal and parietal cortices, a number of ballooned neurons were observed, they were particularly prominent in the cingulate gyrus (Fig. 3a-c). Neuronal loss was most severe in the frontal and parietal cortices, the caudate nucleus and in the CA3 and CA4 subregions of the hippocampus (Fig. 4a). Although neuronal loss was not marked in the dentate fascia of the hippocampus, there was dispersion of the granule cell layer (Fig. 4a). Poorly defined basophilic inclusions were evident in a proportion of neurons in haematoxylin- and eosin-stained sections (Fig. 3d). Tau immunohistochemistry revealed extensive neuronal and glial deposition of phosphorylated tau (AT8, epitope phosphoSer202 and phosphoThr205) (Figs. 3e-k, 4c-h). Neuronal inclusions of various types were observed: crescentic or ring-like perinuclear inclusions; diffuse cytoplasmic staining, sometimes also containing fine filaments; flame-shaped inclusions and globular inclusions. The former were particularly marked in the small neurons of the superficial cortical laminae (Fig. 3f-j). Astrocytic tau pathology was observed with morphological features similar to tufted astrocytes (Fig. 3m, o, q). In white matter there were frequent tau-immunoreactive oligodendroglial inclusions, numerous threads and dots (Fig. 3k). All of these structures were immunoreactive for $4 R$ tau and astrocytic pathology was most clearly visible in this preparation (Figs. 31, m, n; 4b). 3R tau was also detected in a proportion of inclusions, predominantly neuronal, using immunohistochemistry, but immunoreactivity was much more restricted than that of $4 \mathrm{R}$ tau (Fig. 31). AT100-positive staining recognized a proportion of neuronal inclusion and threads. P62 immunohistochemistry highlighted abnormal astrocytes, some of the neuronal inclusions and a proportion of oligodendroglial inclusions in white matter (Fig. 3o). Ubiquitin immunohistochemistry revealed weak immunoreactive inclusions mostly with a morphology compatible with astrocytic inclusions. Gallyas silver staining demonstrated a proportion of neuronal, astrocytic and oligodendroglial inclusions, in addition to some of the thread 

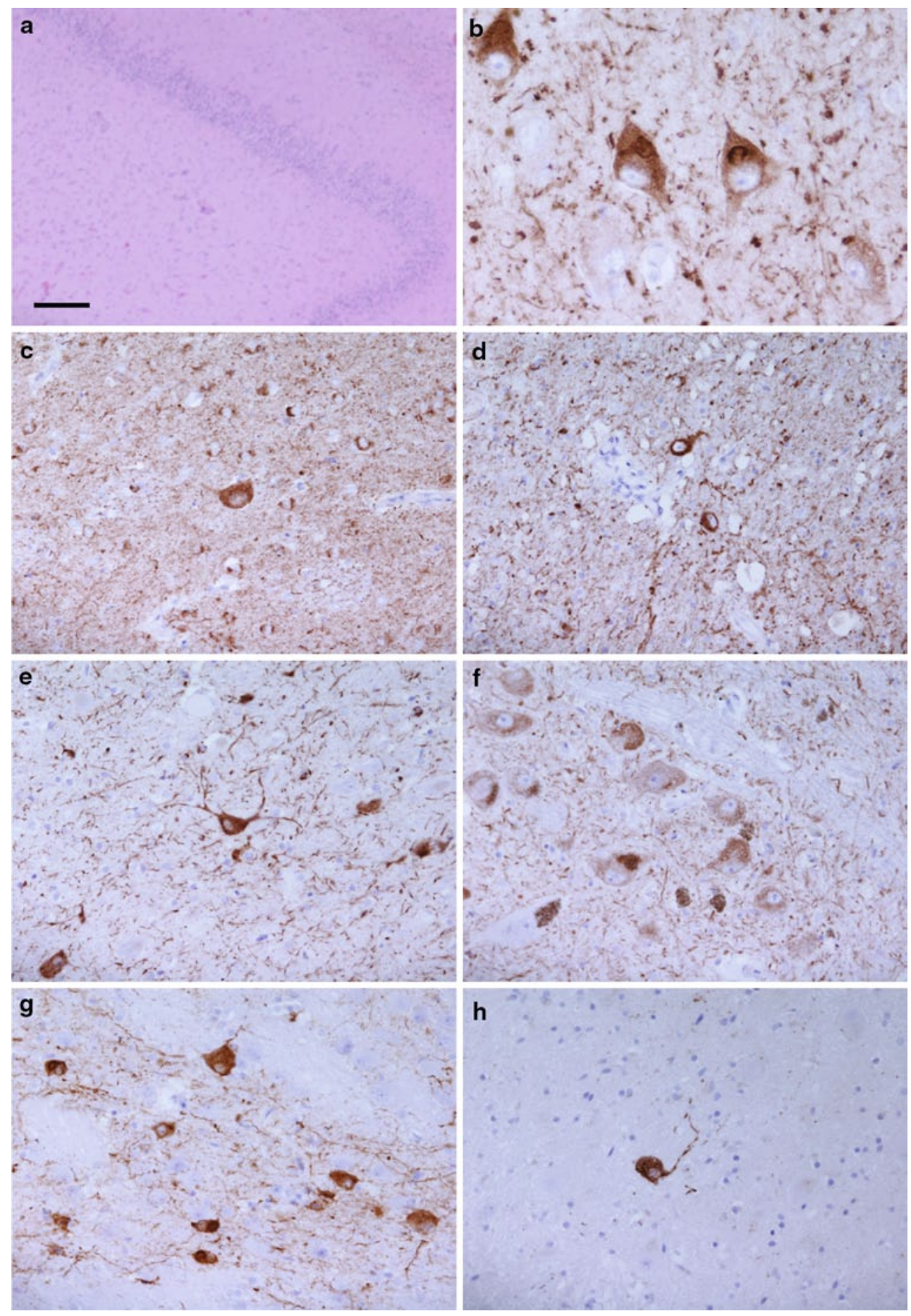

Fig. 4 Neuropathological features in the hippocampus and subcortical nuclei of the proband. In the hippocampal formation there was marked neuronal loss in the CA4 subregion and dispersion of the granule cells of the dentate fascia (a) and tau immunohistochemistry showed widespread neuronal immunoreactivity, often diffuse in character, and sometimes with additional filamentous inclusions (b,

subiculum). Tau-immunoreactive inclusions were widespread in subcortical structures, including: caudate nucleus (c), globus pallidus (d), subthalamic nucleus (e), substantia nigra (f), pontine nuclei (g) and dentate nucleus (h). a H\&E; b 4R tau immunohistochemistry; c-h phospho-tau immunohistochemistry (AT8). Bar in a represents $250 \mu \mathrm{m}$ in $\mathbf{a} ; 50 \mu \mathrm{m}$ in $\mathbf{c}-\mathbf{h} ; 25 \mu \mathrm{m}$ in $\mathbf{b}$

pathology indicating fibrillar tau deposition (Fig. 3p-r). There was no TDP-43 or $\mathrm{A} \beta$ pathology in neocortical regions or the hippocampus. No $\alpha$-synuclein pathology in

the form of Lewy bodies, Lewy neurites or glial cytoplasmic inclusions was found in brainstem, limbic or cortical regions. 
Immunoblotting

To measure the level of expression of tau isoforms, soluble tau was extracted with perchloric acid from the frontal cortex of a patient with the $\mathrm{I} 10+3$ mutation, normal adult human brain and the frontal cortex of the proband with the K298E mutation. All six tau isoforms were expressed in the normal, $\mathrm{I} 10+3$ and $\mathrm{K} 298 \mathrm{E}$ tau mutant brains (Fig. 5a); however, in the K298E case the 4R tau isoforms $1 N 4 R$ appeared increased and the $3 R$ tau isoforms $1 N 3 R$ decreased (Fig. 5a). A slight increase in the 0N4R tau isoform could also be observed in the K298E case. These data showed and confirmed that tau pathology in the frontal cortex had a high prevalence of $4 \mathrm{R}$ tau isoforms. Sarkosyl-insoluble tau extraction from the frontal cortex of the proband with the $\mathrm{K} 298 \mathrm{E} M A P T$ mutation confirmed that $4 \mathrm{R}$ tau was the major component, although some $3 \mathrm{R}$ tau was also present (Fig. 5b). Sarkosyl-insoluble tau extracted from the brain of the $\mathrm{I} 10+3$ case, where filaments had been shown to contain $4 \mathrm{R}$ tau, is shown for comparison.

\section{Tau isoforms expression in K298E-induced neurons}

Direct conversion of human fibroblasts into neurons is of potential clinical utility and of interest for neurological disease modelling, as well as for cell therapeutics [3]. A skin biopsy was taken from the patient with the K298E mutation $2 \mathrm{~h}$ post-mortem and fibroblasts were derived. These were then expanded and converted into iNs, as previously described [24, 25]. As controls, human embryonic fibroblasts (hEFs) and human adult fibroblasts (hAFs) were converted into neurons following an identical protocol. Two weeks after transgene activation, induced neurons started to appear in both controls and K298E cells, but tau was not detected (data not shown). Thirty days after transgene activation, while iNs obtained from human embryonic fibroblasts ( $\mathrm{hEF}-\mathrm{iN}$ ) expressed only the $3 \mathrm{R}$ tau isoform, iNs with the K298E mutation (iN-K298E) and iNs from hAF (hA-iN) expressed both $3 \mathrm{R}$ and $4 \mathrm{R}$ tau (Fig. 6a). Fifty-three days after transgene activation, the level of expression of both tau isoforms in the iN-K298E did not increase (Fig. 6b) and was comparable to the level of tau observed in hAF-iNs. However, hEF-iNs still expressed only 3R tau 53 days after transduction (Fig. 6b). The limited availability of fibroblasts and differentiated neurons did not allow for biochemical studies or more indepth analyses.

\section{Discussion}

We report a novel missense mutation in MAPT in a 67-yearold lady who was referred with dementia and non-fluent aphasia. Genetic analysis revealed the presence of a K298E missense mutation in exon 10 in one of her alleles.

Missense mutations in MAPT have mainly been identified in the coding region, with a cluster of mutations close to the $5^{\prime}$ splice site of exon 10 . Exon 10 mutations and intronic mutations immediately downstream of the $5^{\prime}$ splice site of exon 10 can modulate the splicing of tau pre-mRNA, which leads to an over-representation of 4R tau. However, some exon 10 mutations, including P301L and P301S, only have effects at the protein level $[13,14,20]$. The region of exon 10, which contains Lys 298 has been shown to contain
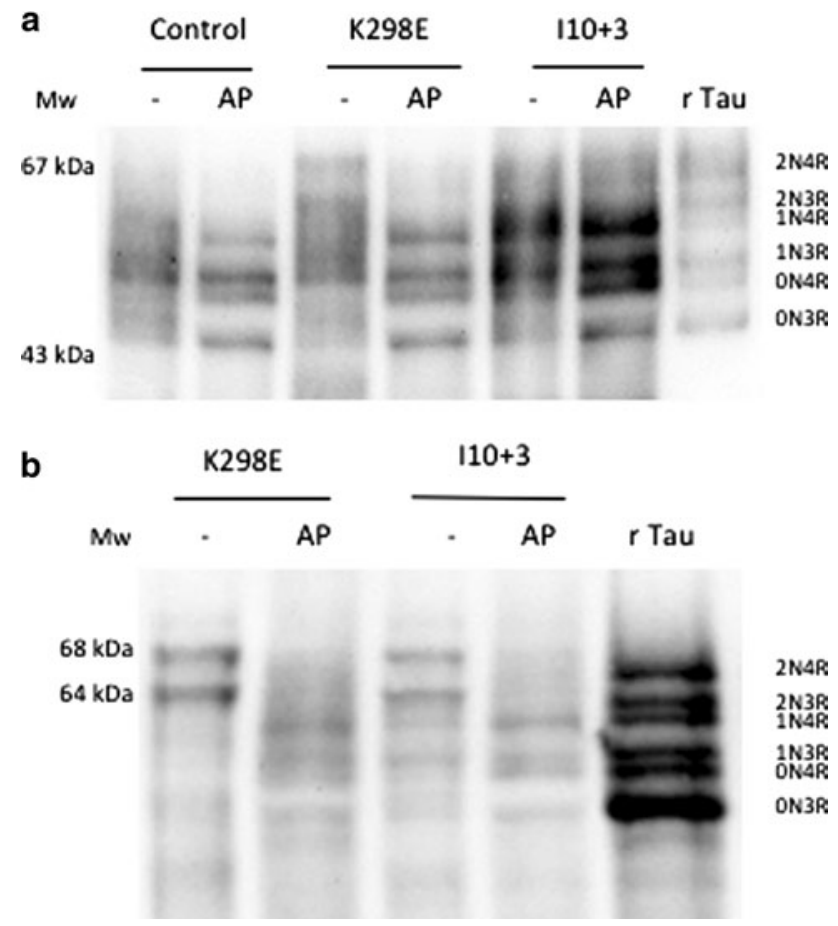

Fig. 5 Soluble and insoluble tau protein from human brain. a Tau isoforms extracted from frontal cortex of brains of the patient with the K298E MAPT mutation, a patient with the I10+3 G>A (+3) MAPT mutation and a control subject before (-) and after (AP) dephosphorylation with alkaline phosphatase (AP). The pattern of tau isoforms extracted from the frontal cortex of the patient with the K298E mutation shows that this mutation induces an increase in $1 \mathrm{~N} 4 \mathrm{R}$ tau isoform expression, with a decrease in $1 \mathrm{~N} 3 \mathrm{R}$ tau isoform. A slight increase can be also observed for the 0N4R tau isoform. As comparison extracts from cortex of a patient with the $\mathrm{I} 10+3 \mathrm{G}>\mathrm{A}(+3)$ MAPT mutation and a control subject before and after AP treatment are also shown. Recombinant human tau (rTau) isoforms were run as a control. Molecular weight markers $\left(M_{\mathrm{w}}\right)$. b Sarkosyl-insoluble tau from the frontal cortex of the patient with the K298E tau mutation before (-) and after (AP) dephosphorylation with alkaline phosphatase and from frontal cortex of a patient with the $\mathrm{I} 10+3 \mathrm{G}>\mathrm{A}(+3)$ mutation. The immunoblot shows that insoluble tau from frontal cortex contained mainly $4 \mathrm{R}$ tau isoforms although some $3 \mathrm{R}$ tau appears also to be present in the K298E case. The extra bands in the +3 extract could be degradation products in a tissue that also contained some Alzheimer type tau changes. Recombinant tau isoforms (rTau) were run in parallel to the samples. Molecular weight markers $\left(M_{\mathrm{w}}\right)$ 
Fig. 6 Tau isoform expression in human K298E-derived neurons. $a 3 R$ and $4 \mathrm{R}$ tau isoform expression in induced neurons with K298E mutation (iN-K298E), in induced neurons from human adult fibroblasts (hAF-iN) and in induced neurons from human embryonic fibroblasts (hEF-iN) at 30 days after transgene activation. b $3 \mathrm{R}$ and $4 \mathrm{R}$ tau isoform expression in iN-K298E, hAF-iN and hEFiN at 53 days post-transgene activation and beginning of differentiation a
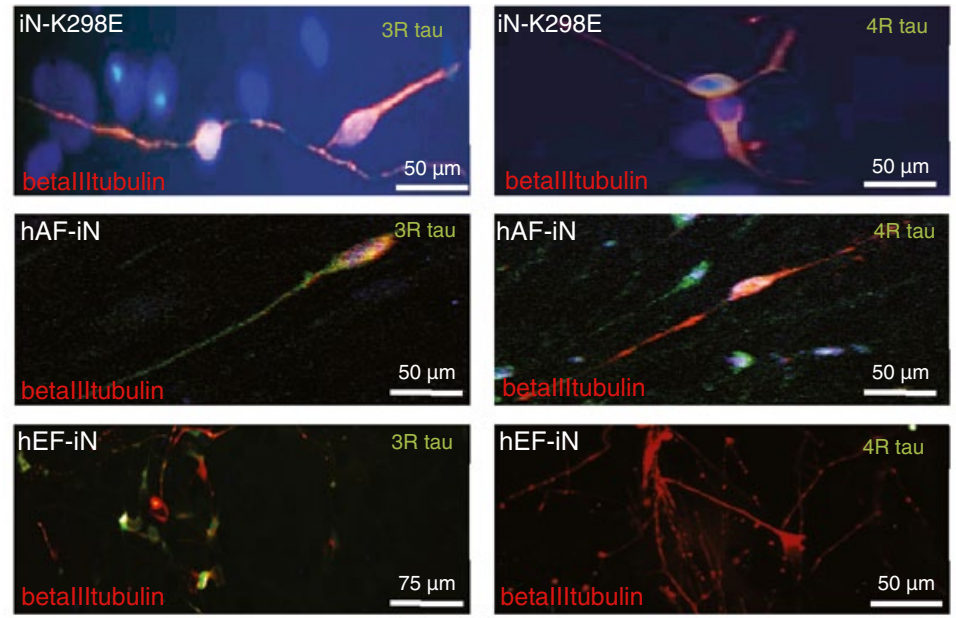

b
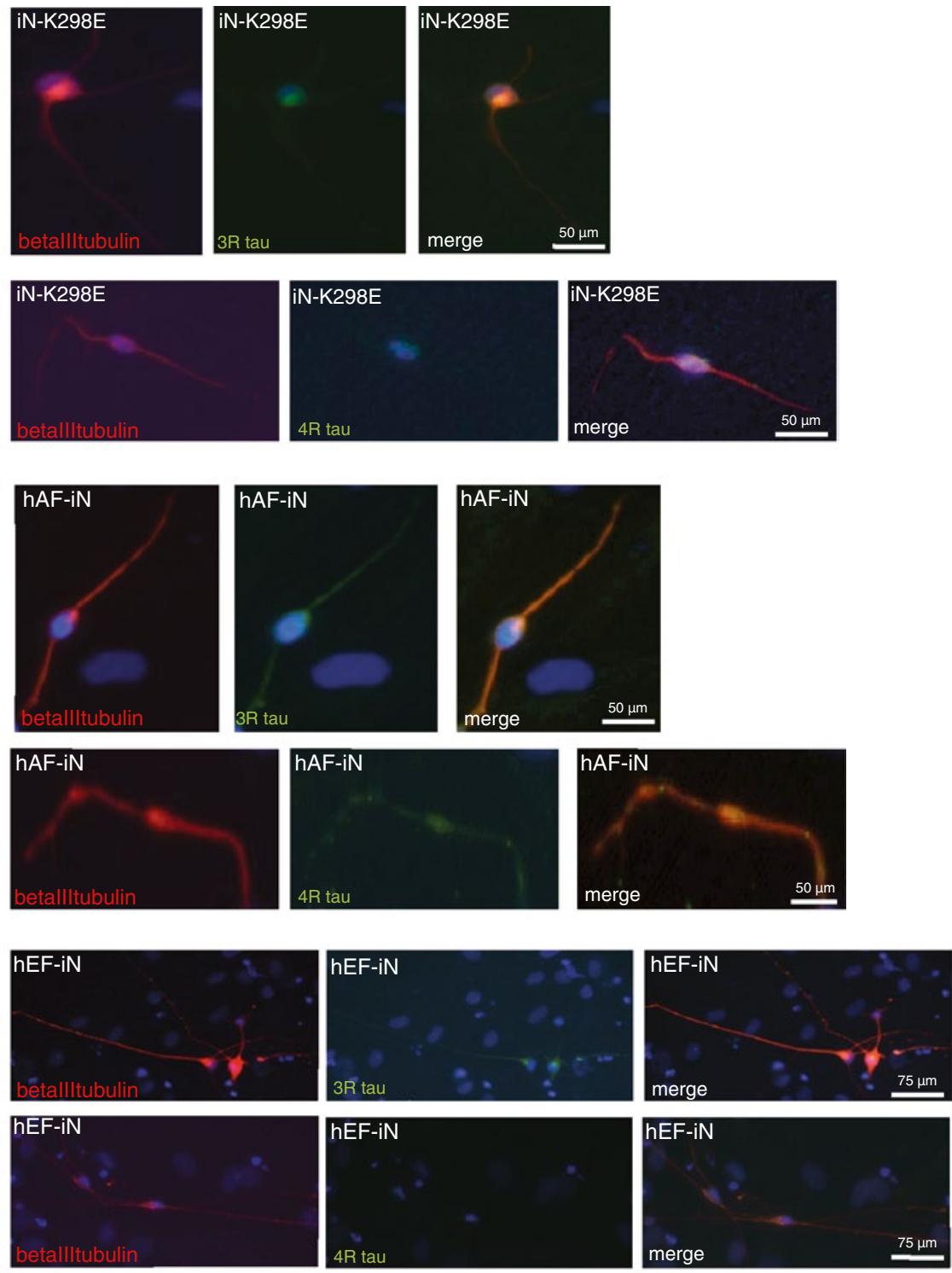

merge 
an exonic splice suppressor (ESS) that negatively regulates the splicing of exon $10[4,5]$. Indeed, mutations at codon 296, in the same ESS, have been described, including $\mathrm{N} 296 \mathrm{H}, \mathrm{N} 296 \mathrm{~N}$, and $\Delta \mathrm{N} 296$ [16, 23, 27]. Interestingly, $\mathrm{N} 296 \mathrm{~N}$ and $\mathrm{N} 296 \mathrm{H}$ lead to an increase in 4R tau mRNA, while mutation $\triangle \mathrm{N} 296$ has no effect on alternative mRNA splicing of MAPT [11, 28]. In contrast, the $\triangle \mathrm{N} 296$ mutation effected a marked reduction in microtubule assembly while the $\mathrm{N} 296 \mathrm{H}$ mutation also significantly reduced microtubule assembly, but less so $\Delta \mathrm{N} 296$ [12]. The K298E mutation similar to the $\mathrm{N} 296 \mathrm{H}$, affects both tau microtubule assembly and $4 \mathrm{R}$ tau expression.

We performed a microtubule assembly assay using mutant K298E recombinant tau protein. The K298E mutation reduced the ability of tau to promote microtubule assembly compared to wild-type tau. We next evaluated the effects of the K298E mutation at the mRNA level and performed minigene transfection experiments. In human NSCs, the transfection of the tau minigene expressing the K298E mutation induced the overexpression of 4R tau (Fig. 2a). Our in vitro data suggest that the K298E mutation induces the overproduction of mutant $4 \mathrm{R}$ tau, which is impaired in its ability to interact with microtubules. The pathogenicity of the K298E mutation was also confirmed by neuropathological analysis of the post-mortem brain, which showed severe atrophy of frontal and anterior temporal lobe, as well as of caudate nucleus and thalamus. Neuronal loss and gliosis confirmed that the pathology was widespread in frontal and parietal cortices, with abundant deposition of hyperphosphorylated tau in neurons and glia, reminiscent of progressive supranuclear palsy [18]. Tau pathology consisted mainly of $4 \mathrm{R}$ tau. Biochemical analysis of soluble tau showed an increase in $4 \mathrm{R}$ and a slight decrease in $3 \mathrm{R}$ tau. In sarkosyl-insoluble tau from frontal cortex of the patient, as in progressive supranuclear palsy, the 64 and $68 \mathrm{kDa}$ bands contained mainly $4 \mathrm{R}$ tau, although a small amount of 3R tau was also present (Fig. 5).

Direct conversion of patient skin fibroblasts into neurons was performed to test whether they express tau isoforms and could show the increase in 4R tau. The skin biopsy was collected $2 \mathrm{~h}$ post-mortem; it was not possible to obtain induced pluripotent stem cells (iPSCs) from the biopsyderived fibroblasts, although several attempts were made by various laboratories. Therefore, we decided to opt for direct conversion of fibroblasts into induced neurons (iNs). Although this approach gave positive results and neurons were produced, they were not in sufficient number to allow biochemical characterization of their tau isoforms. To our knowledge, this is the first study showing tau protein in iNs derived from fibroblasts of a patient with a MAPT mutation collected postmortem. Our analysis has given insight into the study of patient-derived iNs, i.e., that the type of controls used is critical for the evaluation of neuronal phenotype, as far as tau is concerned. We observed a difference in the time course of tau isoform expression between iNs from the proband and iNs from embryonic fibroblasts initially available and used as control, this was not present when comparing iNs from fibroblasts of the proband and of an age-matched control. This suggests that induced neurons obtained from embryonic fibroblasts can take longer to mature compared to those obtained from adult human fibroblasts and are not suitable as control.

In conclusion, we report a novel K298E tau mutation in a patient with frontotemporal dementia. The mutation affected both tau microtubule assembly and tau mRNA splicing with an increase in 4R tau. Therefore, the K298E mutation becomes another member of a small group of exon 10 mutations, which confer pathogenicity through effects at both the RNA and the protein level.

Acknowledgments $\mathrm{MM}$ is funded by a Wellcome Trust Senior Research Fellowship in Clinical Science (101022/Z/13/Z), Parkinson's UK, the Michael J Fox Foundation for Parkinson's disease research, and the pharmaceutical companies supporting the Division of Signal Transduction Therapy Unit (AstraZeneca, BoehringerIngelheim, GlaxoSmithKline, Merck KgA, Janssen Pharmaceutica and Pfizer). JH and TR are supported by Parkinson's UK, the Multiple System Atrophy Trust, Alzheimer's Research UK and the Progressive Supranuclear Palsy (Europe) Association. JH is supported by the Reta Lila Weston Institute for Neurological Studies. TL is supported by Alzheimer's Research UK. The work was partly supported by the National Institute for Health Research (NIHR) Biomedical Research Unit in Dementia based at University College London Hospitals (UCLH), University College London (UCL). The views expressed are those of the author(s) and not necessarily those of the NHS, the NIHR or the Department of Health. MG is supported by the Medical Research Council (U105184291), MGS is supported by the Cambridge Newton Trust, USA Cure PSP, The Scholl Foundation, The Italian cultural association "Il Circolo", Alzheimer's Research UK, Parkinson's UK and the NIHR Cambridge Biomedical Research Centre. Brain tissue from a patient with the $\mathrm{I} 10+3 \mathrm{G}>\mathrm{A}$ MAPT mutation was obtained from the Brain Bank of the Indiana University Alzheimer Centre (US Public Health Service Grant P30 AG010133). Brain tissue from control subject was obtained from the Cambridge Hospital Brain Bank.

Open Access This article is distributed under the terms of the Creative Commons Attribution License which permits any use, distribution, and reproduction in any medium, provided the original author(s) and the source are credited.

\section{References}

1. Anfossi M, Vuono R, Maletta R et al (2011) Compound heterozygosity of 2 novel MAPT mutations in frontotemporal dementia. Neurobiol Aging 32:757.e1-757.e11

2. Brun A (1994) Clinical and neuropathological criteria for frontotemporal dementia. The Lund and Manchester Groups. J Neurol Neurosurg Psychiatry 57:416-418

3. Chambers SM, Studer L (2011) Cell fate plug and play: direct reprogramming and induced pluripotency. Cell 145:827-830

4. D’Souza I, Poorkaj P, Hong M et al (1999) Missense and silent tau gene mutations cause frontotemporal dementia with 
parkinsonism-chromosome 17 type, by affecting multiple alternative RNA splicing regulatory elements. Proc Natl Acad Sci USA 96:5598-5603

5. D'Souza I, Schellenberg GD (2000) Determinants of 4-repeat tau expression. Coordination between enhancing and inhibitory splicing sequences for exon 10 inclusion. J Biol Chem 275:17700-17709

6. Gasparini L, Terni B, Spillantini MG (2007) Frontotemporal dementia with tau pathology. Neurodegener Dis 4:236-253

7. Goedert M, Spillantini MG, Jakes R, Rutherford D, Crowther RA (1989) Multiple isoforms of human microtubule-associated protein tau: sequences and localization in neurofibrillary tangles of Alzheimer's disease. Neuron 3:519-526

8. Goedert M, Jakes R (1990) Expression of separate isoforms of human tau protein: correlation with the tau pattern in brain and effects on tubulin polymerization. EMBO J 9:4225-4230

9. Goedert M, Spillantini MG, Cairns NJ, Crowther RA (1992) Tau proteins of Alzheimer paired helical filaments: abnormal phosphorylation of all 6 brain isoforms. Neuron 8:159-168

10. Goedert M, Ghetti B, Spillantini MG (2012) Frontotemporal dementia: implication for understanding Alzheimer's disease. Cold Spring Harb Perspect Med 2:a006254

11. Grover A, DeTure M, Yen S, Hutton M (2002) Effects on splicing and protein function of three mutations in codon N296 of tau in vitro. Neurosci Lett 323:33-36

12. Gustke N, Trinczek B, Biernat J, Mandelkow EM, Mandelkow E (1994) Domains of tau protein and interaction with microtubules. Biochemistry 33:9511-9522

13. Hasegawa M, Smith MJ, Goedert M (1998) Tau proteins with FTDP-17 mutations have a reduced ability to promote microtubule assembly. FEBS Lett 437:207-210

14. Hong M, Zhukareva V, Vogelsberg-Ragaglia V, Wszolek Z, Reed L, Miller BI, Geschwind DH, Bird TD, McKeel D, Goate A, Morris JC, Wilhelmsen KC, Schellenberg GD, Trojanowski JQ, Lee VM (1998) Mutation-specific functional impairments in distinct tau isoforms of hereditary FTDP-17. Science 282:1914-1917

15. Iovino M, Patani R, Watts C, Chandran S, Spillantini MG (2010) Human stem cell-derived neurons: a system to study human tau function and dysfunction. PLoS One 5:e13947

16. Iseki E, Matsumura T, Marui W et al (2001) Familial frontotemporal dementia and Parkinsonism with a novel N296H mutation in exon 10 of the tau gene and a widespread accumulation of tau in the glial cells. Acta Neuropathol 102:285-292
17. Jiang Z, Cote J, Kwon JM, Goate AM, Wu YJ (2000) Aberrant splicing of tau pre-mRNA caused by intronic mutations associated with the inherited dementia frontotemporal dementia with Parkinsonism linked to chromosome 17. Mol Cell Biol 20:4036-4048

18. Josephs KA, Hodges JR, Snowden JS et al (2011) Neuropathological background of phenotypical variability in frontotemporal dementia. Acta Neuropathol 122:137-153

19. Kumar-Singh S, Van Broeckhoven C (2007) Frontotemporal lobar degeneration: current concepts in the light of recent advances. Brain Pathol 17:104-114

20. Mirra SS, Murrell JR, Gearing M, Spillantini MG, Goedert M, Crowther RA, Levey AI, Jones R, Green J, Shoffner JM, Wainer BH, Schmidt ML, Trojanowski JQ, Ghetti B (1999) Tau pathology in a family with dementia and a P301L mutation in tau. J Neuropathol Exp Neurol 58:335-345

21. Neary D, Snowden JS, Gustafson L et al (1998) Frontotemporal lobar degeneration: a consensus on clinical diagnostic criteria. Neurology 51:1546-1554

22. Onyike CU, Diehl-Schmid J (2013) The epidemiology of frontotemporal dementia. Int Rev Psychiatry 25:130-137

23. Pastor P, Pastor E, Carnero C et al (2001) Familial atypical progressive supranuclear palsy associated with homozygosity for the delN296 mutation in the tau gene. Ann Neurol 49:263-267

24. Patani R, Compston A, Puddifoot CA et al (2009) Activin/Nodal inhibition alone accelerates highly efficient neuronal conversion from human embryonic stem cells and imposes a caudal positional identity. PLoS One 4:e7327

25. Pfisterer U, Kirkeby A, Torper O, Wood J, Nelander J, Dufour A, Björklund A, Lindvall O, Jakobsson J, Parmar M (2011) Direct conversion of human fibroblasts to dopaminergic neurons. Proc Natl Acad Sci USA 108:10343-10348

26. Spillantini MG, Murrell JR, Goedert M, Farlow MR, Klug A, Ghetti B (1998) Mutation in the tau gene in familial multiple system tauopathy with presenile dementia. Proc Natl Acad Sci USA 95:7737-7741

27. Spillantini MG, Yoshida H, Rizzini C et al (2000) A novel tau mutation $(\mathrm{N} 296 \mathrm{~N})$ in familial dementia with swollen achromatic neurons and corticobasal inclusion bodies. Ann Neurol 48:939-943

28. Yoshida H, Crowther RA, Goedert M (2002) Functional effects of tau gene mutations deltaN296 and N296H. J Neurochem 80:548-551 